\title{
A NEW INTEGRAL EQUATION FORMULATION FOR SCATTERING BY PENETRABLE DIFFRACTION GRATINGS*
}

\author{
Ruming Zhang \\ Center for Industrial Mathematics, University of Bremen, Bremen 28359, Germany \\ Email: foeysii@mail.bnu.edu.cn \\ Bo Zhang \\ LSEC and Academy of Mathematics and Systems Science, Chinese Academy of Sciences, Beijing \\ 100190, China and School of Mathematical Sciences, University of Chinese Academy of Sciences, \\ Beijing 10049, China \\ Email: b.zhang@amt.ac.cn
}

\begin{abstract}
This paper is concerned with the problem of scattering of time-harmonic electromagnetic waves from penetrable diffraction gratings in the $2 \mathrm{D}$ polarization case. We propose a new, weakly singular, integral equation formulation for the scattering problem which is proved to be uniquely solvable. A main feature of the new integral equation formulation is that it avoids the computation of the normal derivative of double-layer potentials which is difficult and time consuming. A fast numerical algorithm is also developed for the scattering problem, based on the Nyström method for the new integral equation. Numerical examples are also shown to illustrate the applicability of the new integral equation formulation.
\end{abstract}

Mathematics subject classification: 74J20, 65R20.

Key words: Scattering problem, Transmission condition, Periodic interface, Diffraction gratings, Boundary integral equations, Helmholtz equation.

\section{Introduction}

The integral equation method plays an important role in both the theoretical analysis and the numerical solution of wave scattering problems (see, e.g., [10,11,20]). For example, the integral equation methods have been developed and studied for the scattering problems by bounded obstacles (sound-soft, sound-hard, and impedance cases) in $[10,11,15]$, by periodic structures (called diffraction gratings in the physical and engineering sciences) in $[2,17,18,22,23]$, and by unbounded rough surfaces in $[6-8,25,26]$.

When the scattering obstacles or surfaces are penetrable, the scattering solution satisfies the transmission boundary conditions on the interfaces. In this case, the mostly used integral equations involve combined single- and double-layer boundary operators and their normal derivatives; see, e.g., $[10,12]$ for the bounded obstacle case and $[23,24]$ for the periodic surface case. In [19], several single integral equation formulations were developed for the transmission scattering problem, and in $[9,14]$, the multiple traces boundary integral equation formulations have been proposed for acoustic scattering by composite structures. It is remarked that spectral methods have also been developed in [13] for the periodic case, with the help of the form of

\footnotetext{
* Received January 10, 2016 / Accepted December 5, 2016 /
}

Published online October 11, 2017 / 
quasi-periodic Green's functions. In all the above works, the integral equation formulations contain the hypersingular normal derivatives of double-layer boundary operators which are difficult and time consuming to be computed numerically.

Recently in [1], different from the classical integral equation approach for the scattering problem with a bounded penetrable obstacle, the scattered field is represented by a singlelayer potential, leading to a new integral equation formulation which involves only single-layer boundary operators and their normal derivatives and therefore is weakly singular. The unique solvability of the new integral equation is established in [1] by employing the invertibility of the single-layer boundary operator.

Motivated by this, in this paper, we consider the problem of scattering of time-harmonic plane waves by a $C^{2}$-smooth periodic penetrable interface in $\mathbb{R}^{2}$. We use only single-layer potentials to represent the solution of the scattering problem, and then derive a new boundary integral equation formulation for the scattering problem by using the transmission boundary conditions on the interface. This new integral equation has a simpler form than the classical one and leads to a new, fast numerical method to solve the scattering problem. This numerical method avoids the computation of the normal derivatives of double-layer boundary operators, which occur in the classical boundary integral equation formulations and are difficult and timeconsuming to be computed numerically. This new integral equation is proved to be equivalent to the scattering problem and also uniquely solvable. We will also show by numerical examples that the numerical algorithm based on this new integral equation is convergent.

This paper is organized as follows. In Section 2, we first introduce the scattering problem and then recall its existence and uniqueness results. We study some properties of the singleand double-layer boundary operators in periodic surfaces in Section 3 and review the classical boundary integral equations in Section 4. In Section 5, we present the new integral equation for the scattering problem and prove its equivalence to the scattering problem as well as its unique solvability. In Section 6, we discuss the Nyström method for the new integral equation in detail, and in Section 7, some numerical examples are given to show the convergence of the Nyström method. The final section 8 gives some conclusions.

\section{The Scattering Problem}

Suppose the penetrable diffraction grating $\Gamma$ in $\mathbb{R}^{2}$ is given by

$$
\Gamma=\left\{x \in \mathbb{R}^{2}: x=\left(x_{1}, \gamma\left(x_{1}\right)\right)\right\}
$$

with a $2 \pi$-periodic function $\gamma \in C^{2}$. Then the whole space is divided by $\Gamma$ into two parts:

$$
\Omega_{0}:=\left\{x \in \mathbb{R}^{2}: x_{2}>\gamma\left(x_{1}\right)\right\} \quad \text { and } \Omega_{1}:=\left\{x \in \mathbb{R}^{2}: x_{2}<\gamma\left(x_{1}\right)\right\}
$$

which are filled with different homogeneous materials described by two different refractive indices $k_{0}^{2}>0$ and $k_{1}^{2}>0$, respectively.

Assume that a plane wave, which is given by

$$
u^{i}=\exp \left(i k_{0} x \cdot d\right)=\exp \left[i k_{0}\left(x_{1} \cos \theta-x_{2} \sin \theta\right)\right], \quad \theta \in(0, \pi)
$$

is incident onto the penetrable grating from the top region $\Omega_{0}$, where $d=(\cos \theta,-\sin \theta)$ is the incident direction, $\theta$ is the incident angle, $\alpha=k_{0} \cos \theta$ and $\beta=k_{0} \sin \theta$. Then the problem 
of scattering of time-harmonic electromagnetic waves by the diffraction grating $\Gamma$ in the $2 \mathrm{D}$ polarization case can be modeled by the Helmholtz equations

$$
\begin{array}{ll}
\Delta u+k_{0}^{2} u=0 & \text { in } \Omega_{0} \\
\Delta u+k_{1}^{2} u=0 & \text { in } \Omega_{1}
\end{array}
$$

with the transmission conditions

$$
\left.u\right|_{+}=\left.u\right|_{-} \quad \text { on } \Gamma \quad \text { and }\left.\quad \frac{\partial u}{\partial \nu}\right|_{+}=\left.\lambda \frac{\partial u}{\partial \nu}\right|_{-} \quad \text { on } \Gamma,
$$

where $u$ is the total field which is the sum of the incident wave $u^{i}$ and the scattered wave $u^{s}$ in $\Omega_{0}$, that is, $u=u^{i}+u^{s}$ in $\Omega_{0}, \nu$ is the normal vector at $\Gamma$ directed into $\Omega_{0}, I_{+}$and $\mid$ indicate the limits obtained by approaching the boundary $\Gamma$ from the top domain $\Omega_{0}$ and the bottom domain $\Omega_{1}$, respectively, and $\lambda$ is the transmission constant on the grating $\Gamma$ (note that $\lambda=k_{0}^{2} / k_{1}^{2}$ in the TM-polarization case and $\lambda=1$ in the TE-polarization case). See Figure 2.1 for the physical configuration of the scattering problem.

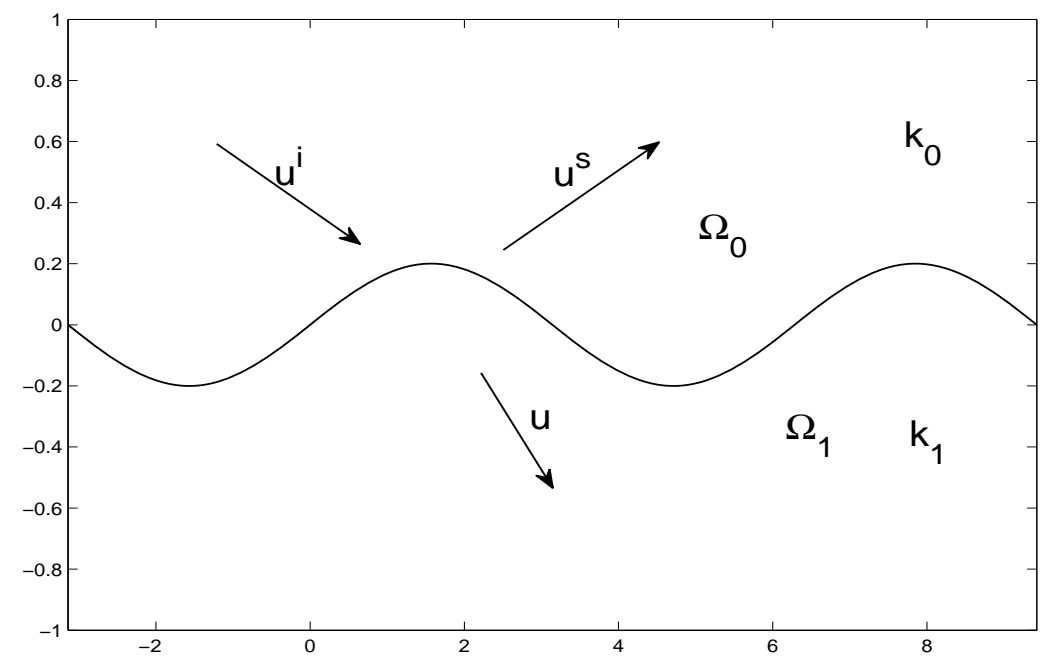

Fig. 2.1. The physical configuration of the scattering problem

In this paper we assume that $\lambda$ satisfies one of the following three conditions: (i) $1<\lambda \leqslant$ $k_{0}^{2} / k_{1}^{2}$, (ii) $1>\lambda \geqslant k_{0}^{2} / k_{1}^{2}$ and (iii) $\lambda=1$. These conditions guarantee the uniqueness of solutions to the above scattering problem (see, e.g., [24]).

Since the incident wave $u^{i}$ is $\alpha$-quasiperiodic in $x_{1}$-direction, that is,

$$
u^{i}\left(x_{1}+2 \pi, x_{2}\right)=\exp (2 i \pi \alpha) u^{i}\left(x_{1}, x_{2}\right) \quad \text { in } \mathbb{R}^{2}
$$

and the grating $\Gamma$ is periodic, the total field $u$ is also required to be $\alpha$-quasiperiodic:

$$
u\left(x_{1}+2 \pi, x_{2}\right)=\exp (2 i \pi \alpha) u\left(x_{1}, x_{2}\right) \quad \text { in } \mathbb{R}^{2} .
$$

Further, the scattered field $u^{s}=u-u^{i}$ in $\Omega_{0}$ and the transmitted field $u$ in $\Omega_{1}$ are assumed to be bounded, which together with the quasi-periodicity of $u$ implies that $u^{s}$ and $u$ satisfy the 
Rayleigh expansion conditions (RECs):

$$
\begin{aligned}
u^{s}(x) & =\sum_{n \in \mathbb{Z}} u_{n}^{(0)} \exp \left\{i \alpha_{n} x_{1}+i \beta_{n}^{(0)} x_{2}\right\}, & & x_{2}>\gamma_{M}:=\max _{t \in \mathbb{R}} \gamma(t) \\
u(x) & =\sum_{n \in \mathbb{Z}} u_{n}^{(1)} \exp \left\{i \alpha_{n} x_{1}-i \beta_{n}^{(1)} x_{2}\right\}, & & x_{2}<\gamma_{m}:=\min _{t \in \mathbb{R}} \gamma(t)
\end{aligned}
$$

with the Rayleigh coefficients $u_{n}^{(j)} \in \mathbb{C}, j=0,1, \alpha_{n}=n+\alpha$ and

$$
\beta_{n}^{(j)}= \begin{cases}\sqrt{k_{j}^{2}-\alpha_{n}^{2}}, & \left|\alpha_{n}\right|<k_{j}, \\ i \sqrt{\alpha_{n}^{2}-k_{j}^{2}}, & \left|\alpha_{n}\right| \geq k_{j}\end{cases}
$$

for $j=0,1$. In this paper, we always assume that $\beta_{n}^{(j)} \neq 0$ for all $n \in \mathbb{Z}, j=0,1$.

We remark that our approach also works for the case when the bottom layer $\Omega_{1}$ may have a complex wavenumber $k_{1}$. In this case, $\beta_{n}^{(1)}$ should be defined as $\beta_{n}^{(1)}=\sqrt{k_{1}^{2}-\alpha_{n}^{2}}$ with the branch cut being taken so that $\operatorname{Re}\left(\beta_{n}^{(1)}\right) \geq 0$ and $\operatorname{Im}\left(\beta_{n}^{(1)}\right) \geq 0$.

For simplicity, hereafter, we use $\Omega_{j}(j=0,1)$ and $\Gamma$ again to denote the same sets restricted to one period $0<x_{1}<2 \pi$.

In this paper, the solution of the scattering problem will be studied in suitable Sobolev spaces. To this end, for any $p \in \mathbb{R}$ we introduce the following Sobolev spaces of $\alpha$-quasi-periodic functions:

$$
\begin{aligned}
& H_{\alpha}^{p}(\Gamma)=\left\{\phi \in H^{p}(\Gamma): \phi \text { is } \alpha \text {-quasi-periodic }\right\}, \\
& H_{\alpha, l o c}^{p}\left(\Omega_{0}\right)=\left\{\phi \in H^{p}\left(\Omega_{0 h}\right) \forall h>\gamma_{M}: \phi \text { is } \alpha \text {-quasi-periodic }\right\}, \\
& H_{\alpha, l o c}^{p}\left(\Omega_{1}\right)=\left\{\phi \in H^{p}\left(\Omega_{1 h}\right) \forall h<\gamma_{m}: \phi \text { is } \alpha \text {-quasi-periodic }\right\},
\end{aligned}
$$

where $\Omega_{0 h}:=\left\{x \in \Omega_{0}: \gamma\left(x_{1}\right)<x_{2}<h\right\}$ for $h>\gamma_{M}, \Omega_{1 h}:=\left\{x \in \Omega_{1}: h<x_{2}<\gamma\left(x_{1}\right)\right\}$ for $h<\gamma_{m}$ and $H^{p}(\Gamma)$ and $H^{p}\left(\Omega_{j h}\right)$ are the standard Sobolev spaces of functions defined on $\Gamma$ and $\Omega_{j h}$, respectively $(j=0,1)$.

Let $v=u^{s}$ in $\Omega_{0}, f=-\left.u^{i}\right|_{\Gamma}, g=-\partial u^{i} /\left.\partial \nu\right|_{\Gamma}$. Then $f \in H_{\alpha}^{1 / 2}(\Gamma), g \in H_{\alpha}^{-1 / 2}(\Gamma)$ and the scattering problem can be rewritten as the transmission problem: For $f \in H_{\alpha}^{1 / 2}(\Gamma)$ and $g \in H_{\alpha}^{-1 / 2}(\Gamma)$, find $v \in H_{\alpha, l o c}^{1}\left(\Omega_{0}\right)$ and $u \in H_{\alpha, l o c}^{1}\left(\Omega_{1}\right)$ such that

$$
\begin{aligned}
& \Delta v+k_{0}^{2} v=0 \quad \text { in } \Omega_{0}, \\
& \Delta u+k_{1}^{2} u=0 \quad \text { in } \Omega_{1} \\
& \left.v\right|_{+}-\left.u\right|_{-}=f \quad \text { on } \Gamma \\
& \left.\frac{\partial u}{\partial \nu}\right|_{+}-\left.\lambda \frac{\partial u}{\partial \nu}\right|_{-}=g \text { on } \Gamma \\
& v, u \text { satisfy }(2.2) \text { and }(2.3), \text { respectively. }
\end{aligned}
$$

Arguing similarly as in the proof of Theorem 2.2 in [24], we can obtain the following uniqueness and existence results of solutions to the transmission problem (2.5)-(2.9).

Theorem 2.1. Assume that $\beta_{n}^{(j)} \neq 0$ for all $n \in \mathbb{Z}, j=0,1$. For any $(f, g) \in H_{\alpha}^{1 / 2}(\Gamma) \times$ $H_{\alpha}^{-1 / 2}(\Gamma)$, the transmission problem $(2.5)-(2.9)$ has a unique solution $(v, u) \in H_{\alpha, l o c}^{1}\left(\Omega_{0}\right) \times$ $H_{\alpha, l o c}^{1}\left(\Omega_{1}\right)$ depending continuously on the boundary data. 


\section{Layer Operators on Periodic Surfaces}

We now study properties of single- and double-layer operators on the periodic surface $\Gamma$. To this end, for arbitrarily fixed wave number $k>0$ let $\Phi(x, y)=(i / 2) H_{0}^{(1)}(k|x-y|), \quad x \neq y$, and let

$$
G(x, y)=\frac{i}{4 \pi} \sum_{n \in \mathbb{Z}} \frac{1}{\beta_{n}} \exp \left[i \alpha_{n}\left(x_{1}-y_{1}\right)+i \beta_{n}\left|x_{2}-y_{2}\right|\right], \quad x \neq y
$$

with $\beta_{n} \neq 0$, where $\beta_{n}$ is defined as $\beta_{n}^{(0)}$ in $(2.4)$ with $k_{0}$ replaced by $k$. Then $\Phi$ and $G$ are the free-space Green's function and the free-space $\alpha$-quasiperiodic Green's function in $\mathbb{R}^{2} \backslash\{(2 n \pi, 0): n \in \mathbb{Z}\}$ of the Helmholtz equation $\Delta w+k^{2} w=0$, respectively. Note that $a(x, y):=G(x, y)-\Phi(x, y)$ is analytic in $[(-\pi, \pi) \times \mathbb{R}] \times[(-\pi, \pi) \times \mathbb{R}]$ (see $[17])$.

Define the single- and double-layer operators:

$$
\begin{array}{ll}
(S \phi)(x)=\int_{\Gamma} G(x, y) \phi(y) d s(y), & \phi \in H_{\alpha}^{-1 / 2}(\Gamma), \\
(K \phi)(x)=\int_{\Gamma} \frac{\partial G(x, y)}{\partial \nu(y)} \phi(y) d s(y), & \phi \in H_{\alpha}^{1 / 2}(\Gamma),
\end{array}
$$

and their normal derivatives:

$$
\begin{array}{ll}
\left(K^{\prime} \phi\right)(x)=\int_{\Gamma} \frac{\partial G(x, y)}{\partial \nu(x)} \phi(y) d s(y), & \phi \in H_{\alpha}^{-1 / 2}(\Gamma), \\
(T \phi)(x)=\frac{\partial}{\partial \nu(x)} \int_{\Gamma} \frac{\partial G(x, y)}{\partial \nu(y)} \phi(y) d s(y), & \phi \in H_{\alpha}^{1 / 2}(\Gamma) .
\end{array}
$$

The properties of the layer operators $S, K$ and $K^{\prime}$ are similar to the corresponding operators defined on a closed smooth curve with the kernel $\Phi(x, y)$, so we have the following two theorems (cf. Theorems 8.22 and 8.25 in [20]).

Theorem 3.1. The operators $K: H_{\alpha}^{1 / 2}(\Gamma) \rightarrow H_{\alpha}^{1 / 2}(\Gamma)$ and $K^{\prime}: H_{\alpha}^{-1 / 2}(\Gamma) \rightarrow H_{\alpha}^{-1 / 2}(\Gamma)$ are compact and adjoint with respect to the dual system $<\cdot, \cdot\rangle_{L^{2}(\Gamma)}$, that is,

$$
<K \phi, \psi>_{L^{2}(\Gamma)}=<\phi, K^{\prime} \psi>_{L^{2}(\Gamma)}
$$

for all $\phi \in H_{\alpha}^{1 / 2}(\Gamma)$ and $\psi \in H_{\alpha}^{-1 / 2}(\Gamma)$.

Theorem 3.2. The operator $S: H_{\alpha}^{-1 / 2}(\Gamma) \rightarrow H_{\alpha}^{1 / 2}(\Gamma)$ is bounded and self-adjoint with respect to the dual system $\langle\cdot, \cdot\rangle_{L^{2}(\Gamma)}$, that is,

$$
<S \phi, \psi>_{L^{2}(\Gamma)}=<\phi, S \psi>_{L^{2}(\Gamma)}
$$

for all $\phi, \psi \in H_{\alpha}^{-1 / 2}(\Gamma)$.

For the single-layer boundary operator defined on a closed smooth curve $C$ with the kernel $\Phi(x, y)$, it is well known that it is invertible in the Sobolev space $H^{-1 / 2}(C)$ if $k$ is not an interior Dirichlet eigenvalue (see [10] for a proof). However, the single-layer boundary operator $S$ defined by (3.1) on the periodic curve $\Gamma$ has a better property that it is invertible in the Sobolev space $H_{\alpha}^{-1 / 2}(\Gamma)$ for all wave numbers $k$ satisfying $\beta_{n} \neq 0$ for all $n \in \mathbb{Z}$, since no Dirichlet eigenvalues exist in either the upper domain $\Omega_{0}$ or the lower domain $\Omega_{1}$, as presented in the following theorem. 
Theorem 3.3. The single-layer boundary operator $S: H_{\alpha}^{-1 / 2}(\Gamma) \rightarrow H_{\alpha}^{1 / 2}(\Gamma)$ is invertible with its inverse $S^{-1}$ being a bounded linear operator from $H_{\alpha}^{1 / 2}(\Gamma)$ to $H_{\alpha}^{-1 / 2}(\Gamma)$.

To prove Theorem 3.3, we need the following result on the well-posedness of the boundary value problem in $\Omega_{0}$ or $\Omega_{1}$ with a Dirichlet boundary condition on $\Gamma$. This result is proved in the classical spaces in [17] (see Theorem 5 in [17]) and can be easily extended to the case of Sobolev spaces.

Lemma 3.1. For any $f \in H_{\alpha}^{1 / 2}(\Gamma)$ the boundary value problem

$$
\left\{\begin{array}{l}
\text { Find } w \in H_{\alpha, l o c}^{1}\left(\Omega_{j}\right) \text { such that } \\
\Delta w+k^{2} w=0 \text { in } \Omega_{j}, \quad w=f \text { on } \Gamma \\
w \text { satisfies }(2.2) \text { with } j=0 \text { or }(2.3) \text { with } j=1
\end{array}\right.
$$

has a unique solution $w$ which depends continuously on the boundary data, where $j=0,1$. Further, the normal derivative $\partial w / \partial \nu$ on $\Gamma$ satisfies the estimate

$$
\left\|\frac{\partial w}{\partial \nu}\right\|_{H^{-1 / 2}(\Gamma)} \leq C\|f\|_{H^{1 / 2}(\Gamma)} .
$$

We now prove Theorem 3.3 with the help of Lemma 3.1.

Proof of Theorem 3.3. We first prove that $S$ is injective. Assume that $S \phi=0$ for some $\phi \in H_{\alpha}^{-1 / 2}(\Gamma)$. Define

$$
w(x)=\int_{\Gamma} G(x, y) \phi(y) d s(y), \quad x \in \mathbb{R}_{\pi}^{2} \backslash \Gamma,
$$

where $\mathbb{R}_{\pi}^{2}:=\left\{x \in \mathbb{R}^{2}: 0<x_{1}<2 \pi\right\}$. Then $w$ is $\alpha$-quasiperiodic and satisfies the Helmholtz equation $\Delta w+k^{2} w=0$ in $\Omega_{j}(j=0,1)$ and the RECs (2.2) and (2.3). By the jump property of the single-layer potential, we have $\left.w\right|_{+}=\left.w\right|_{-}=S \phi=0$ on $\Gamma$. Thus, by Lemma $3.1 w=0$ in $\Omega_{j}$ with $j=0,1$. By the jump property of the single-layer potential again we have

$$
0=\left.\frac{\partial w}{\partial \nu}\right|_{+}-\left.\frac{\partial w}{\partial \nu}\right|_{-}=-2 \phi \quad \text { on } \Gamma
$$

Then the operator $S$ is injective, so its inverse $S^{-1}$ exists.

We now show that $S^{-1}$ is a bounded linear operator from $H_{\alpha}^{1 / 2}(\Gamma)$ to $H_{\alpha}^{-1 / 2}(\Gamma)$. To this end, we prove that for any $f \in H_{\alpha}^{1 / 2}(\Gamma)$ there exists a function $\phi \in H_{\alpha}^{-1 / 2}(\Gamma)$ such that $S \phi=f$ with $\phi$ depending continuously on $f$.

By Lemma 3.1, for any $f \in H_{\alpha}^{1 / 2}(\Gamma)$ there exists a unique function $w_{j} \in H_{\alpha, l o c}^{1}\left(\Omega_{j}\right)$ such that $\Delta w_{j}+k^{2} w_{j}=0$ in $\Omega_{j}$ and $w_{j}=f$ on $\Gamma$, where $w_{j}$ satisfies (2.2) with $j=0$ or (2.3) with $j=1$, where $j=0,1$. By Lemma 3.1 again, the normal derivative $\partial w_{j} / \partial \nu \in H_{\alpha}^{-1 / 2}(\Gamma)$ satisfies

$$
\left\|\frac{\partial w_{j}}{\partial \nu}\right\|_{H^{-1 / 2}(\Gamma)} \leq C\|f\|_{H^{1 / 2}(\Gamma)}, \quad j=0,1
$$

From Green's formula we have

$$
\begin{array}{ll}
2 w_{0}(x)=\int_{\Gamma}\left[\left.\frac{\partial G(x, y)}{\partial \nu(y)} w_{0}(y)\right|_{+}-\left.G(x, y) \frac{\partial w_{0}(y)}{\partial \nu(y)}\right|_{+}\right] d s(y), \quad & x \in \Omega_{0}, \\
2 w_{1}(x)=\int_{\Gamma}\left[-\left.\frac{\partial G(x, y)}{\partial \nu(y)} w_{1}(y)\right|_{-}+\left.G(x, y) \frac{\partial w_{1}(y)}{\partial \nu(y)}\right|_{-}\right] d s(y), & x \in \Omega_{1} .
\end{array}
$$


Then, letting $x \rightarrow \Gamma$ from $\Omega_{0}$ and $\Omega_{1}$, respectively, we have

$$
\begin{aligned}
& 2 f=\left.2 w_{0}\right|_{\Gamma}=(K+I) f-\left.S \frac{\partial w_{0}}{\partial \nu}\right|_{\Gamma}, \\
& 2 f=\left.2 w_{1}\right|_{\Gamma}=-(K-I) f+\left.S \frac{\partial w_{1}}{\partial \nu}\right|_{\Gamma} .
\end{aligned}
$$

Summing up the above two equations gives

$$
2 f=S\left(\left.\frac{\partial w_{1}}{\partial \nu}\right|_{\Gamma}-\left.\frac{\partial w_{0}}{\partial \nu}\right|_{\Gamma}\right) .
$$

Define

$$
\phi=\frac{1}{2}\left(\left.\frac{\partial w_{1}}{\partial \nu}\right|_{\Gamma}-\left.\frac{\partial w_{0}}{\partial \nu}\right|_{\Gamma}\right)
$$

Then $\phi \in H_{\alpha}^{-1 / 2}(\Gamma)$ satisfies that $S \phi=f$ and

$$
\|\phi\|_{H^{-1 / 2}(\Gamma)} \leq \frac{1}{2}\left(\left\|\frac{\partial w_{1}}{\partial \nu}\right\|_{H^{-1 / 2}(\Gamma)}+\left\|\frac{\partial w_{0}}{\partial \nu}\right\|_{H^{-1 / 2}(\Gamma)}\right) \leq C\|f\|_{H^{1 / 2}(\Gamma)} .
$$

The proof is thus completed.

The invertibility of $S$ plays a key role in deriving our new integral equation.

\section{Classical Boundary Integral Equations}

We now review the classical formulation of boundary integral equations for the scattering problem (2.5)-(2.9). Seek a solution $(v, u) \in H_{\alpha, l o c}^{1}\left(\Omega_{0}\right) \times H_{\alpha, l o c}^{1}\left(\Omega_{1}\right)$ of the scattering problem (2.5)-(2.9) in the form

$$
\begin{array}{lll}
v(x)=\int_{\Gamma} G_{0}(x, y) \psi_{0}(y) d s(y)+\lambda \int_{\Gamma} \frac{\partial G_{0}(x, y)}{\partial \nu(y)} \psi_{1}(y) d s(y), & x \in \Omega_{0}, \\
u(x)=\int_{\Gamma} G_{1}(x, y) \psi_{0}(y) d s(y)+\int_{\Gamma} \frac{\partial G_{1}(x, y)}{\partial \nu(y)} \psi_{1}(y) d s(y), & x \in \Omega_{1},
\end{array}
$$

where $\psi_{0} \in H_{\alpha}^{-1 / 2}(\Gamma), \psi_{1} \in H_{\alpha}^{1 / 2}(\Gamma)$, and $G_{j}(x, y)$ stands for the quasi-periodic Green's function defined as $G$ with $k$ replaced by $k_{j}, j=0,1$. Then this leads to the classical boundary integral equation:

$$
\left(\begin{array}{cc}
S_{0}-S_{1} & \lambda K_{0}-K_{1}+(\lambda+1) I \\
K_{0}^{\prime}-\lambda K_{1}^{\prime}-(\lambda+1) I & \lambda\left(T_{0}-T_{1}\right)
\end{array}\right)\left(\begin{array}{l}
\psi_{0} \\
\psi_{1}
\end{array}\right)=\left(\begin{array}{l}
f \\
g
\end{array}\right) .
$$

Here, $S_{j}, K_{j}, K_{j}^{\prime}$, and $T_{j}$ are defined as $S, K, K^{\prime}$, and $T$ with $G$ replaced by $G_{j}, j=0,1$. It was proved in [24] that the integral equation (4.3) is uniquely solvable.

This kind of integral equations is widely used in the numerical solutions of the scattering problems by bounded penetrable obstacles (see, e.g., $[12,16,19]$ ). The numerical discretization of single- and double-layer boundary operators is discussed in [20], and that of their normal derivatives is presented in [21]. The integral equation (4.3) contains two single-layer boundary operators $S_{j}$, two double-layer boundary operators $K_{j}$, and their normal derivatives $K_{j}^{\prime}$ and $T_{j}$, $j=0,1$. In these integral operators, $T_{0}$ and $T_{1}$ are hypersingular operators whose numerical computation is very difficult and time-consuming.

In the next section, we will derive a new boundary integral equation which only involves single-layer boundary operators and their normal derivatives whose numerical computation is easier and faster. 


\section{A New Integral Equation Formulation}

In this section, we will propose a new boundary integral equation formulation for the scattering problem (2.5)-(2.9) and prove its equivalency to the scattering problem as well as its unique solvability.

We now seek a solution $(v, u) \in H_{\alpha, l o c}^{1}\left(\Omega_{0}\right) \times H_{\alpha, l o c}^{1}\left(\Omega_{1}\right)$ of the scattering problem (2.5)-(2.9) in the form

$$
\begin{array}{ll}
v(x)=\int_{\Gamma} G_{0}(x, y) \phi_{0}(y) d s(y), & x \in \Omega_{0}, \\
u(x)=\int_{\Gamma} G_{1}(x, y) \phi_{1}(y) d s(y), & x \in \Omega_{1},
\end{array}
$$

where $\phi_{0}, \phi_{1} \in H_{\alpha}^{-1 / 2}(\Gamma)$. Then $v$ and $u$ are $\alpha$-quasiperiodic and satisfy the Helmholtz equations (2.5) and (2.6) and the Rayleigh expansion conditions (2.2) and (2.3), respectively. From the jump properties of the single-layer potentials and their derivatives, the Cauchy data of $v$ and $u$ on $\Gamma$ can be written in the forms:

$$
\begin{array}{ll}
\left.v\right|_{+}=S_{0} \phi_{0}, & \left.\frac{\partial v}{\partial \nu}\right|_{+}=\left(K_{0}^{\prime}-I\right) \phi_{0}, \\
\left.u\right|_{-}=S_{1} \phi_{1}, & \left.\frac{\partial u}{\partial \nu}\right|_{-}=\left(K_{1}^{\prime}+I\right) \phi_{1} .
\end{array}
$$

From the transmission boundary conditions (2.7)-(2.8), we arrive at the following integral equation formulation:

$$
\left(\begin{array}{cc}
S_{0} & -S_{1} \\
K_{0}^{\prime}-I & -\lambda K_{1}^{\prime}-\lambda I
\end{array}\right)\left(\begin{array}{c}
\phi_{0} \\
\phi_{1}
\end{array}\right)=\left(\begin{array}{c}
f \\
g
\end{array}\right) .
$$

We now show that the scattering problem (2.5)-(2.9) is equivalent to the integral equation (5.3).

Theorem 5.1. If $(v, u) \in H_{\alpha, l o c}^{1}\left(\Omega_{0}\right) \times H_{\alpha, l o c}^{1}\left(\Omega_{1}\right)$ is a solution of the scattering problem (2.5)(2.9), then there exists $\left(\phi_{0}, \phi_{1}\right) \in H_{\alpha}^{-1 / 2}(\Gamma) \times H_{\alpha}^{-1 / 2}(\Gamma)$ satisfying the integral equation (5.3), and $v$ and $u$ can be represented in terms of $\phi_{0}$ and $\phi_{1}$ in the form of (5.1)-(5.2). Conversely, if $\left(\phi_{0}, \phi_{1}\right) \in H_{\alpha}^{-1 / 2}(\Gamma) \times H_{\alpha}^{-1 / 2}(\Gamma)$ satisfies the integral equation (5.3) and $v$ and $u$ are defined by (5.1)-(5.2), then $(v, u) \in H_{\alpha, l o c}^{1}\left(\Omega_{0}\right) \times H_{\alpha, l o c}^{1}\left(\Omega_{1}\right)$ is a solution of the scattering problem (2.5)-(2.9)

Proof. First, assume that $(v, u) \in H_{\alpha, l o c}^{1}\left(\Omega_{0}\right) \times H_{\alpha, l o c}^{1}\left(\Omega_{1}\right)$ satisfies (2.5)-(2.9). Then $\left.v\right|_{+},\left.u\right|_{-} \in H_{\alpha}^{1 / 2}(\Gamma)$ and $\partial v /\left.\partial \nu\right|_{+}, \partial u /\left.\partial \nu\right|_{-} \in H_{\alpha}^{-1 / 2}(\Gamma)$. From Green's theorem, it follows that $v$ and $u$ satisfy the following equations:

$$
\begin{array}{ll}
2 v(x)=\int_{\Gamma}\left[\left.\frac{\partial G_{0}(x, y)}{\partial \nu(y)} v(y)\right|_{+}-\left.G_{0}(x, y) \frac{\partial v(y)}{\partial \nu(y)}\right|_{+}\right] d s(y), & x \in \Omega_{0}, \\
2 u(x)=\int_{\Gamma}\left[-\left.\frac{\partial G_{1}(x, y)}{\partial \nu(y)} u(y)\right|_{-}+\left.G_{1}(x, y) \frac{\partial u(y)}{\partial \nu(y)}\right|_{-}\right] d s(y), & x \in \Omega_{1} .
\end{array}
$$

Letting $x$ approach $\Gamma$ from $\Omega_{0}$ and $\Omega_{1}$, respectively, we have

$$
\begin{aligned}
& \left.2 v\right|_{+}=\left.\left(K_{0}+I\right) v\right|_{+}-\left.S_{0} \frac{\partial v}{\partial \nu}\right|_{+} \\
& \left.2 u\right|_{-}=-\left.\left(K_{1}-I\right) u\right|_{-}+\left.S_{1} \frac{\partial u}{\partial \nu}\right|_{-},
\end{aligned}
$$


that is,

$$
\left.v\right|_{+}=\left.K_{0} v\right|_{+}-\left.S_{0} \frac{\partial v}{\partial \nu}\right|_{+},\left.\quad u\right|_{-}=-\left.K_{1} u\right|_{-}+\left.S_{1} \frac{\partial u}{\partial \nu}\right|_{-} .
$$

Define

$$
\phi_{0}=\left.S_{0}^{-1} K_{0} v\right|_{+}-\left.\frac{\partial v}{\partial \nu}\right|_{+}, \quad \phi_{1}=-\left.S_{1}^{-1} K_{1} u\right|_{-}+\left.\frac{\partial u}{\partial \nu}\right|_{-} .
$$

Then $\phi_{0}, \phi_{1} \in H_{\alpha}^{-1 / 2}(\Gamma)$ and satisfy that $\left.v\right|_{+}=S_{0} \phi_{0}$ and $\left.u\right|_{-}=S_{1} \phi_{1}$ on $\Gamma$. Let

$$
\tilde{v}(x)=\int_{\Gamma} G_{0}(x, y) \phi_{0}(y) d s(y), \quad \tilde{u}(x)=\int_{\Gamma} G_{1}(x, y) \phi_{1}(y) d s(y) .
$$

Then $\left.\tilde{v}\right|_{+}=\left.v\right|_{+}=S_{0} \phi_{0}$ on $\Gamma$. Since $\tilde{v}$ satisfies the Helmholtz equation (2.5) and the Rayleigh expansion condition (2.2), then we have by Lemma 3.1 that $\tilde{v}=v$ in $\Omega_{0}$. Similarly, we have $\tilde{u}=u$ in $\Omega_{1}$. Then $v$ and $u$ can be written in the form of (5.1) and (5.2). Eq. (5.3) follows directly from the jump properties of layer potentials and the transmission boundary conditions (2.7)-(2.8).

Conversely, if $\left(\phi_{0}, \phi_{1}\right) \in H_{\alpha}^{-1 / 2}(\Gamma) \times H_{\alpha}^{-1 / 2}(\Gamma)$ satisfies (5.3) and $v, u$ are defined by (5.1)(5.2), then it is straightforward to verify that $(v, u)$ satisfies $(2.5)-(2.9)$.

Denote by $A$ the matrix operator in (5.3). Then $A$ is a linear operator from the space $H_{\alpha}^{-1 / 2}(\Gamma) \times H_{\alpha}^{-1 / 2}(\Gamma)$ to $H_{\alpha}^{1 / 2}(\Gamma) \times H_{\alpha}^{-1 / 2}(\Gamma)$. Further, $A$ is invertible with a bounded inverse. We first show that $A$ is injective.

Theorem 5.2. Assume that $\beta_{n}^{(j)} \neq 0$ for all $n \in \mathbb{Z}, j=0,1$. Then $A: H_{\alpha}^{-1 / 2}(\Gamma) \times H_{\alpha}^{-1 / 2}(\Gamma) \rightarrow$ $H_{\alpha}^{1 / 2}(\Gamma) \times H_{\alpha}^{-1 / 2}(\Gamma)$ is injective.

Proof. Suppose there exist $\phi_{0}, \phi_{1} \in H_{\alpha}^{-1 / 2}(\Gamma)$ such that

$$
A\left(\begin{array}{c}
\phi_{0} \\
\phi_{1}
\end{array}\right)=\left(\begin{array}{l}
0 \\
0
\end{array}\right) \text {. }
$$

Define

$$
\begin{aligned}
& v(x):=\int_{\Gamma} G_{0}(x, y) \phi_{0}(y) d s(y), x \in \Omega_{0} \\
& u(x):=\int_{\Gamma} G_{1}(x, y) \phi_{1}(y) d s(y), \quad x \in \Omega_{1}
\end{aligned}
$$

Then $\left(\left.v\right|_{\Omega_{0}},\left.u\right|_{\Omega_{1}}\right)$ satisfies the problem (2.5)-(2.9) with $f=g=0$. Then it follows from Theorem 2.1 that $v=0$ in $\Omega_{0}$ and $u=0$ in $\Omega_{1}$. By the continuity of the single-layer potentials, we obtain that $\left.v\right|_{+}=S_{0} \phi_{0}=0$ and $\left.u\right|_{-}=S_{1} \phi_{1}=0$. By Theorem 3.2, $\phi_{0}=0, \phi_{1}=0$. This means that $A$ is injective, and the proof is thus complete.

We have shown that $A$ is injective, so its inverse $A^{-1}$ exists. In fact, $A^{-1}$ is also bounded, as proved in the following result.

Theorem 5.3. Assume that $\beta_{n}^{(j)} \neq 0$ for all $n \in \mathbb{Z}, j=0,1$. Then $A$ is invertible, and its inverse $A^{-1}$ is a bounded operator from $H_{\alpha}^{1 / 2}(\Gamma) \times H_{\alpha}^{-1 / 2}(\Gamma)$ to $H_{\alpha}^{-1 / 2}(\Gamma) \times H_{\alpha}^{-1 / 2}(\Gamma)$. 
Proof. Define the operator $B$ as

$$
B=\left(\begin{array}{cc}
S_{0}^{-1} & 0 \\
0 & I
\end{array}\right) A=\left(\begin{array}{cc}
I & -S_{0}^{-1} S_{1} \\
K_{0}^{\prime}-I & -\lambda K_{1}^{\prime}-\lambda I
\end{array}\right)
$$

Then $B$ is an operator from the space $H_{\alpha}^{-1 / 2}(\Gamma) \times H_{\alpha}^{-1 / 2}(\Gamma)$ to itself. By Theorem $5.2 A$ is injective, so $B$ is also injective. Write $B=B_{1}+B_{2}$ with

$$
\begin{aligned}
& B_{1}=\left(\begin{array}{cc}
I & -S_{0}^{-1} S_{1} \\
-I & -(\lambda+1) I+S_{0}^{-1} S_{1}
\end{array}\right), \\
& B_{2}=\left(\begin{array}{cc}
0 & 0 \\
K_{0}^{\prime} & -\lambda K_{1}^{\prime}-S_{0}^{-1}\left(S_{1}-S_{0}\right)
\end{array}\right) .
\end{aligned}
$$

It is easy to prove that $B_{1}$ is invertible with its inverse

$$
B_{1}^{-1}=\frac{1}{\lambda+1}\left(\begin{array}{cc}
(\lambda+1) I-S_{0}^{-1} S_{1} & -S_{0}^{-1} S_{1} \\
-I & -I
\end{array}\right)
$$

We now consider $B_{2}$. By Theorem $3.1 K_{0}^{\prime}$ and $K_{1}^{\prime}$ are compact operators from $H_{\alpha}^{-1 / 2}(\Gamma)$ to $H_{\alpha}^{-1 / 2}(\Gamma)$. Arguing similarly as in the proof of Lemma 2.1 in [5] for the case of a closed Lipschitz surface $\Gamma$, we can obtain that $S_{1}-S_{0}$ is compact from $H_{\alpha}^{-1 / 2}(\Gamma)$ to $H_{\alpha}^{12}(\Gamma)$. Thus, by the boundedness of $S_{0}^{-1}, S_{0}^{-1}\left(S_{1}-S_{0}\right)$ is compact from $H_{\alpha}^{-1 / 2}(\Gamma)$ to $H_{\alpha}^{-1 / 2}(\Gamma)$. This implies that $B_{2}$ is compact. Therefore, $B$ is a Fredholm operator with index zero, so, by the Fredholm alternative $B$ is invertible with the inverse $B^{-1}$ being bounded from $H_{\alpha}^{-1 / 2}(\Gamma) \times H_{\alpha}^{-1 / 2}(\Gamma)$ to $H_{\alpha}^{-1 / 2}(\Gamma) \times H_{\alpha}^{-1 / 2}(\Gamma)$. Hence,

$$
A=\left(\begin{array}{cc}
S_{0} & 0 \\
0 & I
\end{array}\right) B
$$

is invertible, and its inverse

$$
A^{-1}=B^{-1}\left(\begin{array}{cc}
S_{0}^{-1} & 0 \\
0 & I
\end{array}\right)
$$

is bounded from $H_{\alpha}^{1 / 2}(\Gamma) \times H_{\alpha}^{-1 / 2}(\Gamma)$ to $H_{\alpha}^{-1 / 2}(\Gamma) \times H_{\alpha}^{-1 / 2}(\Gamma)$.

Theorem 5.3 implies that the integral equation (5.3) is uniquely solvable in $H_{\alpha}^{1 / 2}(\Gamma) \times$ $H_{\alpha}^{-1 / 2}(\Gamma)$. In the next section, we develop a fast Nyström method to solve the integral equation (5.3).

\section{The Nyström Method}

We now use the Nyström method to solve the integral equation (5.3). The Nyström method was presented in [11] for a weakly singular integral equation of the second kind over a closed smooth curve. The method is a little different for the periodic curve case.

Suppose the points on $\Gamma$ have the parametric representation of the following forms:

$$
x(t)=(t, \gamma(t)), \quad 0 \leqslant t \leqslant 2 \pi,
$$


and let $y=x(\tau)$. Then the boundary integral operators (3.1) and (3.3) can be written as

$$
\begin{aligned}
& (S \phi)(x(t))=\int_{0}^{2 \pi} G(x(t), x(\tau)) \phi(x(\tau)) \sqrt{1+\left[\gamma^{\prime}(\tau)\right]^{2}} d \tau \\
& =e^{i \alpha t} \int_{0}^{2 \pi} G(x(t), x(\tau)) e^{-i \alpha(t-\tau)} \phi(x(\tau)) e^{-i \alpha \tau} \sqrt{1+\left[\gamma^{\prime}(\tau)\right]^{2}} d \tau \\
& \left(K^{\prime} \phi\right)(x(t))=\int_{0}^{2 \pi}\left(-\gamma^{\prime}(t), 1\right) \cdot \nabla G(x(t), x(\tau)) \phi(x(\tau)) \sqrt{\frac{1+\left[\gamma^{\prime}(\tau)\right]^{2}}{1+\left[\gamma^{\prime}(t)\right]^{2}}} d \tau \\
& \quad=e^{i \alpha t} \int_{0}^{2 \pi}\left(-\gamma^{\prime}(t), 1\right) \cdot \nabla G(x(t), x(\tau)) e^{-i \alpha(t-\tau)} \phi(x(\tau)) e^{-i \alpha \tau} \sqrt{\frac{1+\left[\gamma^{\prime}(\tau)\right]^{2}}{1+\left[\gamma^{\prime}(t)\right]^{2}}} d \tau .
\end{aligned}
$$

Write $\mathcal{S}(t)=(S \phi)(x(t)) e^{-i \alpha t}, \mathcal{K}(t)=\left(K^{\prime} \phi\right)(x(t)) e^{-i \alpha t}$ and $\psi(\tau)=\phi(x(\tau)) e^{-i \alpha \tau}$. Then $\psi$ is periodic, and the integral operators $\mathcal{S}(t)$ and $\mathcal{K}(t)$ have the following forms

$$
\begin{aligned}
& \mathcal{S}(t)=\int_{0}^{2 \pi} G(x(t), x(\tau)) e^{-i \alpha(t-\tau)} \sqrt{1+\left[\gamma^{\prime}(\tau)\right]^{2}} \psi(\tau) d \tau \\
& \mathcal{K}(t)=\int_{0}^{2 \pi}\left(-\gamma^{\prime}(t), 1\right) \cdot \nabla G(x(t), x(\tau)) e^{-i \alpha(t-\tau)} \sqrt{\frac{1+\left[\gamma^{\prime}(\tau)\right]^{2}}{1+\left[\gamma^{\prime}(t)\right]^{2}}} \psi(\tau) d \tau .
\end{aligned}
$$

Since $G(x, y)$ is $\alpha$-quasi-periodic, $G(x(t), x(\tau)) e^{-i \alpha(t-\tau)}$ and $\nabla G(x(t), x(\tau)) e^{-i \alpha(t-\tau)}$ are periodic. So the integrands of $\mathcal{S}(t)$ and $\mathcal{K}(t)$ are $2 \pi$-periodic with respect to $t$ and $\tau$. Thus, the integration interval $[0,2 \pi]$ can be translated into $[t-\tau, t+\tau]$. Define the transformation $\mathscr{F}$ by

$$
\mathscr{F}(\tau ; t)=\tau+2 \pi\left[\frac{t-\tau}{2 \pi}+\frac{1}{2}\right],
$$

where the square brackets mean rounding towards minus infinity. Then the interval $[0,2 \pi]$ is transformed into $[t-\pi, t+\pi]$ by $\mathscr{F}$. The purpose of this transformation is to move the singularity at $\tau=t$ to the middle of the integration interval for convenience when dealing with the singular integration. So the integral operators $\mathcal{S}(t)$ and $\mathcal{K}(t)$ can be written in the following forms:

$$
\begin{aligned}
& \mathcal{S}(t)=\int_{0}^{2 \pi} G(x(t), x(\mathscr{F}(\tau ; t))) e^{-i \alpha(t-\mathscr{F}(\tau ; t))} \sqrt{1+\left[\gamma^{\prime}(\tau)\right]^{2}} \psi(\tau) d \tau \\
& \mathcal{K}(t)=\int_{0}^{2 \pi}\left(-\gamma^{\prime}(t), 1\right) \cdot \nabla G(x(t), x(\mathscr{F}(\tau ; t))) e^{-i \alpha(t-\mathscr{F}(\tau ; t))} \sqrt{\frac{1+\left[\gamma^{\prime}(\tau)\right]^{2}}{1+\left[\gamma^{\prime}(t)\right]^{2}}} \psi(\tau) d \tau .
\end{aligned}
$$

The quasi-periodic Green's function $G$ is defined in terms of an infinite series which converges very slowly, so its numerical computation is time consuming, especially for large wave numbers $k$. Many efficient numerical methods have been developed to compute the quasi-periodic Green's functions. Recently, we have developed a fast FFT-based algorithm for efficient computation of the quasi-periodic Green's functions [27], which will be used in this paper. To this end, we rewrite the Green's function and its gradient as the sum of a regular and a singular functions:

$$
\begin{aligned}
& G(x, y) e^{-i \alpha\left(x_{1}-y_{1}\right)}=g_{1}(x, y)+h_{1}(x-y), \\
& \nabla G(x, y) e^{-i \alpha\left(x_{1}-y_{1}\right)}=g_{2}(x, y)+h_{2}(x-y)(x-y),
\end{aligned}
$$


where $g_{1}, g_{2}$ are $C^{\mu}$ functions $(0<\mu<2)$ and $h_{1}, h_{2}$ are singular functions with the singularity at 0 . The singular functions $h_{1}$ and $h_{2}$ are defined by

$$
\begin{aligned}
& h_{1}(x)=\frac{\mathcal{X}\left(x_{1}\right)}{2 \pi}\left(1-i \alpha x_{1}\right) \ln r \\
& h_{2}(x)=\frac{\mathcal{X}\left(x_{1}\right)}{2 \pi}\left[\frac{1-i \alpha x_{1}-\frac{1}{2} \alpha^{2} x_{1}^{2}}{r^{2}}-\frac{k^{2}}{2} \ln r\right],
\end{aligned}
$$

where $x=\left(x_{1}, x_{2}\right), r=\sqrt{x_{1}^{2}+x_{2}^{2}}$ and $\mathcal{X}(r)$ is a smooth cutoff function supported in $[-1,1]$. Let

$$
\begin{aligned}
& \mathcal{G}_{1}(t, \tau)=g_{1}(x(t), x(\mathscr{F}(\tau ; t))) \sqrt{1+\left[\gamma^{\prime}(\tau)\right]^{2}}, \\
& \mathcal{G}_{2}(t, \tau)=\left(-\gamma^{\prime}(t), 1\right) \cdot g_{2}(x(t), x(\mathscr{F}(\tau ; t))) \sqrt{\frac{1+\left[\gamma^{\prime}(\tau)\right]^{2}}{1+\left[\gamma^{\prime}(t)\right]^{2}}}, \\
& \mathcal{H}_{1}(t, \tau)=h_{1}(x(t)-x(\mathscr{F}(\tau ; t))) \sqrt{1+\left[\gamma^{\prime}(\tau)\right]^{2}}, \\
& \mathcal{H}_{2}(t, \tau)=\left(-\gamma^{\prime}(t), 1\right) \cdot(x(t)-x(\mathscr{F}(\tau ; t))) h_{2}(x(t)-x(\mathscr{F}(\tau ; t))) \sqrt{\frac{1+\left[\gamma^{\prime}(\tau)\right]^{2}}{1+\left[\gamma^{\prime}(t)\right]^{2}}},
\end{aligned}
$$

Then we have

$$
\begin{aligned}
& \mathcal{S}(t)=\int_{0}^{2 \pi} \mathcal{G}_{1}(t, \tau) \psi(\tau) d \tau+\int_{0}^{2 \pi} \mathcal{H}_{1}(t, \tau) \psi(\tau) d \tau \\
& \mathcal{K}(t)=\int_{0}^{2 \pi} \mathcal{G}_{2}(t, \tau) \psi(\tau) d \tau+\int_{0}^{2 \pi} \mathcal{H}_{2}(t, \tau) \psi(\tau) d \tau .
\end{aligned}
$$

The kernel functions $\mathcal{G}_{1}$ and $\mathcal{G}_{2}$ are regular, so the first terms can be calculated by the classical quadrature rules. Now consider the second terms. Let $r(t, \tau)=\sqrt{(t-\tau)^{2}+(\gamma(t)-\gamma(\tau))^{2}}$. Then, similarly as in [11], we split the kernels up into the two sums:

$$
\begin{aligned}
& \mathcal{H}_{1}(t, \tau)=M_{1}(t, \tau) \ln \left(4 \sin ^{2} \frac{t-\mathscr{F}(\tau ; t)}{2}\right)+M_{2}(t, \tau), \\
& \mathcal{H}_{2}(t, \tau)=L_{1}(t, \tau) \ln \left(4 \sin ^{2} \frac{t-\mathscr{F}(\tau ; t)}{2}\right)+L_{2}(t, \tau),
\end{aligned}
$$

where

$$
\begin{aligned}
& M_{1}(t, \tau)=-\frac{1}{2 \pi} J_{0}(k r(t, \mathscr{F}(\tau ; t))) \sqrt{1+\left[\gamma^{\prime}(\tau)\right]^{2}} \mathcal{X}(t-\mathscr{F}(\tau ; t)), \\
& M_{2}(t, \tau)=\mathcal{H}_{1}(t, \tau)-M_{1}(t, \tau) \ln \left(4 \sin ^{2} \frac{t-\mathscr{F}(\tau ; t)}{2}\right), \\
& L_{1}(t, \tau)=\frac{k}{2 \pi} \frac{J_{1}(k r(t, \mathscr{F}(\tau ; t)))}{r(t, \mathscr{F}(\tau ; t))}\{[\gamma(t)-\gamma(\mathscr{F}(\tau ; t))] \\
& \left.-\gamma^{\prime}(t)[t-\mathscr{F}(\tau ; t)]\right\} \sqrt{\frac{1+\left[\gamma^{\prime}(\tau)\right]^{2}}{1+\left[\gamma^{\prime}(t)\right]^{2}}} \mathcal{X}(t-\mathscr{F}(\tau ; t)), \\
& L_{2}(t, \tau)=\mathcal{H}_{2}(t, \tau)-L_{1}(t, \tau) \ln \left(4 \sin ^{2} \frac{t-\mathscr{F}(\tau ; t)}{2}\right) \text {, }
\end{aligned}
$$


with

$$
\begin{aligned}
& M_{2}(t, t)=(2 \pi)^{-1} \sqrt{1+\left[\gamma^{\prime}(t)\right]^{2}} \ln \sqrt{1+\left[\gamma^{\prime}(t)\right]^{2}}, \\
& L_{2}(t, t)=-\gamma^{\prime \prime}(t) /\left[4 \pi\left(1+\left[\gamma^{\prime}(t)\right]^{2}\right)\right] .
\end{aligned}
$$

To apply the Nyström method, the functions $M_{1}$ and $L_{1}$ are required to be periodic. To this end, we need to introduce a cutoff function into the representation of these two functions. Choose the set of knots: $t_{j}=(j / n) \pi, j=0, \ldots, 2 n-1$, and use the quadrature rule

$$
\int_{0}^{2 \pi} \ln \left(4 \sin ^{2} \frac{t-\tau}{2}\right) f(\tau) d \tau \approx \sum_{j=0}^{2 n-1} R_{j}^{n}(t) f\left(t_{j}\right), \quad 0 \leqslant t \leqslant 2 \pi
$$

with the quadrature weights given by

$$
R_{j}^{n}(t)=-\frac{2 \pi}{n} \sum_{m=1}^{n-1} \frac{1}{m} \cos m\left(t-t_{j}\right)-\frac{\pi}{n^{2}} \cos n\left(t-t_{j}\right), \quad j=0, \ldots, 2 n-1
$$

and the trapezoidal rule

$$
\int_{0}^{2 \pi} f(\tau) d \tau \approx \frac{\pi}{n} \sum_{j=0}^{2 n-1} f\left(t_{j}\right)
$$

Then the integral operators $\mathcal{S}(t)$ and $\mathcal{K}(t)$ can be approximated as follows:

$$
\begin{aligned}
& \mathcal{S}(t) \approx \sum_{j=0}^{2 n-1}\left\{R_{j}^{n}(t) M_{1}\left(t, t_{j}\right)+\frac{\pi}{n}\left(\mathcal{G}_{1}\left(t, t_{j}\right)+M_{2}\left(t, t_{j}\right)\right)\right\} \psi\left(t_{j}\right), \\
& \mathcal{K}(t) \approx \sum_{j=0}^{2 n-1}\left\{R_{j}^{n}(t) L_{1}\left(t, t_{j}\right)+\frac{\pi}{n}\left(\mathcal{G}_{2}\left(t, t_{j}\right)+L_{2}\left(t, t_{j}\right)\right)\right\} \psi\left(t_{j}\right) .
\end{aligned}
$$

Let $r_{j}^{n}=R_{j}^{n}(0)$. For the points $t_{l}, l=0, \ldots, 2 n-1$, we have

$$
\begin{aligned}
R_{j}^{n}\left(t_{l}\right) & =-\frac{2 \pi}{n} \sum_{m=1}^{n-1} \frac{1}{m} \cos m\left(t_{l}-t_{j}\right)-\frac{\pi}{n^{2}} \cos n\left(t_{l}-t_{j}\right) \\
& =-\frac{2 \pi}{n} \sum_{m=1}^{n-1} \frac{1}{m} \cos m \frac{\pi(l-j)}{n}-\frac{\pi}{n^{2}} \cos n \frac{\pi(l-j)}{n}=R_{|l-j|}^{n}(0) .
\end{aligned}
$$

Let $r_{|l-j|}^{n}=R_{|l-j|}^{n}(0)$ and let

$$
\begin{aligned}
& P(l, j)=r_{|l-j|}^{n} M_{1}\left(t_{l}, t_{j}\right)+\frac{\pi}{n}\left(\mathcal{G}_{1}\left(t_{l}, t_{j}\right)+M_{2}\left(t_{l}, t_{j}\right)\right), \\
& Q(l, j)=r_{|l-j|}^{n} L_{1}\left(t_{l}, t_{j}\right)+\frac{\pi}{n}\left(\mathcal{G}_{2}\left(t_{l}, t_{j}\right)+L_{2}\left(t_{l}, t_{j}\right)\right) .
\end{aligned}
$$

Then

$$
\begin{aligned}
& \mathcal{S}\left(t_{l}\right) \approx \sum_{j=0}^{2 n-1} P(l, j) \psi_{j}^{(n)}, \\
& \mathcal{K}\left(t_{l}\right) \approx \sum_{j=0}^{2 n-1} Q(l, j) \psi_{j}^{(n)} .
\end{aligned}
$$


We now apply the above discretization method to the integral equation (5.3). Multiply the integral equation (5.3) by the factor $e^{-i \alpha t}$ and define $\psi_{j}(t)=e^{-i \alpha t} \phi_{j}(x(t)), j=0,1$, $\hat{f}(t)=e^{-i \alpha t} f(x(t))$ and $\hat{g}(t)=e^{-i \alpha t} g(x(t))$. Then the integral equation (5.3) can be rewritten as

$$
\left(\begin{array}{cc}
\mathcal{S}_{0} & -\mathcal{S}_{1} \\
\mathcal{K}_{0}-I & -\lambda \mathcal{K}_{1}-\lambda I
\end{array}\right)\left(\begin{array}{c}
\psi_{0} \\
\psi_{1}
\end{array}\right)=\left(\begin{array}{l}
\hat{f} \\
\hat{g}
\end{array}\right)
$$

Denote $\psi_{0 j}^{(n)}=\psi_{0}\left(t_{j}\right), \psi_{1 j}^{(n)}=\psi_{1}\left(t_{j}\right), f_{l}^{(n)}=\hat{f}\left(t_{l}\right)$ and $g_{l}^{(n)}=\hat{g}\left(t_{l}\right)$. Apply the discretization formulas (6.1) and (6.2) to the integral operators $\mathcal{S}_{m}$ and $\mathcal{K}_{m}$, respectively, and denote by $P^{m}(l, j)$ and $Q^{m}(l, j)$ the corresponding kernels $P(l, j)$ and $Q(l, j)$, respectively, with $m=0,1$. Then the discrete form of the integral equation (6.3) becomes

$$
\begin{aligned}
& \sum_{j=0}^{2 n-1} P^{0}(l, j) \psi_{0 j}^{(n)}-\sum_{j=0}^{2 n-1} P^{1}(l, j) \psi_{1 j}^{(n)}=f_{l}^{(n)}, \\
& \sum_{j=0}^{2 n-1} \tilde{Q}^{0}(l, j) \psi_{0 j}^{(n)}-\sum_{j=0}^{2 n-1} \tilde{Q}^{1}(l, j) \psi_{1 j}^{(n)}=g_{l}^{(n)},
\end{aligned}
$$

where

$$
\begin{aligned}
& \tilde{Q}^{0}(l, j)= \begin{cases}Q^{0}(l, j), & \text { if } l \neq j, \\
Q^{0}(l, j)-1, & \text { if } l=j,\end{cases} \\
& \tilde{Q}^{1}(l, j)= \begin{cases}\lambda Q^{1}(l, j), & \text { if } l \neq j, \\
\lambda Q^{1}(l, j)+\lambda, & \text { if } l=j .\end{cases}
\end{aligned}
$$

The linear system (6.4)-(6.5) can be solved by many numerical methods, such as the GMRES method, the conjugate gradient method and the multi-grid methods.

\section{Numerical Results}

In this section, we will present some numerical examples to show that the Nyström method derived from the new integral equation (5.3) is effective.

We first present two examples for a flat interface, that is, $\Gamma=\Gamma_{0}:=\left\{x \in \mathbb{R}^{2}: x=\left(x_{1}, 1\right)\right\}$, to show the convergence of our numerical scheme. In this case, if the incident field is a plane wave, that is, $u^{i}(x)=\exp \left(i \alpha x_{1}-i \beta_{0}^{(0)} x_{2}\right)$, then the scattered field $u^{s}$ and the total field $u$ have the following explicit presentations:

$$
\begin{aligned}
& u^{s}(x)=\frac{\beta_{0}^{(0)}-\lambda \beta_{0}^{(1)}}{\beta_{0}^{(0)}+\lambda \beta_{0}^{(1)}} \exp \left(-2 i \beta_{0}^{(0)}\right) \exp \left(i \alpha x_{1}+i \beta_{0}^{(0)} x_{2}\right), \\
& u(x)=\frac{2 \beta_{0}^{(0)}}{\beta_{0}^{(0)}+\lambda \beta_{0}^{(1)}} \exp \left(i \beta_{0}^{(1)}-i \beta_{0}^{(0)}\right) \exp \left(i \alpha x_{1}-i \beta_{0}^{(1)} x_{2}\right) .
\end{aligned}
$$

The first example is for the TM-polarization case with $k_{0}=2, k_{1}=4, \lambda=k_{0}^{2} / k_{1}^{2}$ and the incident angle $\theta=\frac{\pi}{4}$, and the second one is for the TE-polarization case with $k_{0}=50, k_{1}=$ 
Table 7.1: Numerical results for the flat profile $\left(\lambda=k_{0}^{2} / k_{1}^{2}\right): k_{0}=2, k_{1}=4, \theta=\frac{\pi}{4}, \Gamma=\Gamma_{0}$.

\begin{tabular}{|c|c|c|c|c|}
\hline$N$ & $u\left(P_{1}\right)$ & $u\left(P_{2}\right)$ & $u\left(P_{3}\right)$ & $u\left(P_{4}\right)$ \\
\hline 4 & $-0.018516-0.822593 \mathrm{i}$ & $-0.787970+0.236868 \mathrm{i}$ & $1.014435-0.649189 \mathrm{i}$ & $-0.895853-0.804967 \mathrm{i}$ \\
\hline 8 & $-0.053822-0.807661 \mathrm{i}$ & $-0.764176+0.266914 \mathrm{i}$ & $0.979253-0.700087 \mathrm{i}$ & $-0.935547-0.757503 \mathrm{i}$ \\
\hline 16 & $-0.055667-0.806757 \mathrm{i}$ & $-0.762813+0.268461 \mathrm{i}$ & $0.977450-0.702617 \mathrm{i}$ & $-0.937505-0.755091 \mathrm{i}$ \\
\hline 32 & $-0.055873-0.806655 \mathrm{i}$ & $-0.762660+0.268632 \mathrm{i}$ & $0.977247-0.702900 \mathrm{i}$ & $-0.937723-0.754820 \mathrm{i}$ \\
\hline 64 & $-0.055898-0.806642 \mathrm{i}$ & $-0.762642+0.268653 \mathrm{i}$ & $0.977222-0.702933 \mathrm{i}$ & $-0.937749-0.754788 \mathrm{i}$ \\
\hline 128 & $-0.055901-0.806641 \mathrm{i}$ & $-0.762640+0.268655 \mathrm{i}$ & $0.977220-0.702937 \mathrm{i}$ & $-0.937752-0.754784 \mathrm{i}$ \\
\hline exact & $-0.055902-0.806641 \mathrm{i}$ & $-0.762638+0.268657 \mathrm{i}$ & $0.977218-0.702939 \mathrm{i}$ & $-0.937754-0.754782 \mathrm{i}$ \\
\hline
\end{tabular}

Table 7.2: Numerical results for the flat profile $(\lambda=1): k_{0}=50, k_{1}=50 \sqrt{3}, \theta=\frac{\pi}{90}, \Gamma=\Gamma_{0}$.

\begin{tabular}{|c|c|c|c|c|}
\hline$N$ & $u\left(P_{1}\right)$ & $u\left(P_{2}\right)$ & $u\left(P_{3}\right)$ & $u\left(P_{4}\right)$ \\
\hline 128 & $-0.384212-1.883127 \mathrm{i}$ & $-0.562373-1.837804 \mathrm{i}$ & $-0.009491-0.047208 \mathrm{i}$ & $-0.013958-0.046085 \mathrm{i}$ \\
\hline 256 & $-0.386321-1.883080 \mathrm{i}$ & $-0.564469-1.837556 \mathrm{i}$ & $-0.009542-0.047198 \mathrm{i}$ & $-0.014008-0.046070 \mathrm{i}$ \\
\hline 512 & $-0.386509-1.883076 \mathrm{i}$ & $-0.564655-1.837533 \mathrm{i}$ & $-0.009546-0.047197 \mathrm{i}$ & $-0.014012-0.046069 \mathrm{i}$ \\
\hline 1024 & $-0.386508-1.883076 \mathrm{i}$ & $-0.564654-1.837534 \mathrm{i}$ & $-0.009546-0.047197 \mathrm{i}$ & $-0.014012-0.046069 \mathrm{i}$ \\
\hline exact & $-0.386508-1.883076 \mathrm{i}$ & $-0.564654-1.837534 \mathrm{i}$ & $-0.009546-0.047197 \mathrm{i}$ & $-0.014012-0.046069 \mathrm{i}$ \\
\hline
\end{tabular}

Table 7.3: Numerical results for the TM case $\left(\lambda=k_{0}^{2} / k_{1}^{2}\right): k_{0}=2, k_{1}=4, \theta=\frac{\pi}{4}, \Gamma=\Gamma_{1}$.

\begin{tabular}{|c|c|c|c|c|}
\hline$N$ & $u\left(P_{1}\right)$ & $u\left(P_{2}\right)$ & $u\left(P_{3}\right)$ & $u\left(P_{4}\right)$ \\
\hline 4 & $-0.984914-0.399884 \mathrm{i}$ & $-0.948620-0.323269 \mathrm{i}$ & $-0.547915+0.531961 \mathrm{i}$ & $-0.251525+0.651509 \mathrm{i}$ \\
\hline 8 & $-1.068199-0.289020 \mathrm{i}$ & $-1.060215-0.280058 \mathrm{i}$ & $-0.761333+0.537676 \mathrm{i}$ & $-0.416556+0.745354 \mathrm{i}$ \\
\hline 16 & $-1.068553-0.289523 \mathrm{i}$ & $-1.056802-0.280541 \mathrm{i}$ & $-0.774413+0.542352 \mathrm{i}$ & $-0.44005+0.740624 \mathrm{i}$ \\
\hline 32 & $-1.068559-0.289337 \mathrm{i}$ & $-1.056790-0.280364 \mathrm{i}$ & $-0.774233+0.542594 \mathrm{i}$ & $-0.439990+0.740606 \mathrm{i}$ \\
\hline 64 & $-1.068560-0.289315 \mathrm{i}$ & $-1.056789-0.280343 \mathrm{i}$ & $-0.774212+0.542621 \mathrm{i}$ & $-0.439982+0.740603 \mathrm{i}$ \\
\hline 128 & $-1.068560-0.289312 \mathrm{i}$ & $-1.056789-0.280340 \mathrm{i}$ & $-0.774209+0.542624 \mathrm{i}$ & $-0.439981+0.740603 \mathrm{i}$ \\
\hline
\end{tabular}

Table 7.4: Numerical results for the TM case $\left(\lambda=k_{0}^{2} / k_{1}^{2}\right): k_{0}=2, k_{1}=4, \theta=\frac{\pi}{90}, \Gamma=\Gamma_{1}$.

\begin{tabular}{|c|c|c|c|c|}
\hline$N$ & $u\left(P_{1}\right)$ & $u\left(P_{2}\right)$ & $u\left(P_{3}\right)$ & $u\left(P_{4}\right)$ \\
\hline 4 & $0.114214-0.131731 \mathrm{i}$ & $0.111317-0.122239 \mathrm{i}$ & $-0.050224-0.040273 \mathrm{i}$ & $-0.032345-0.005530 \mathrm{i}$ \\
\hline 8 & $0.096391-0.134704 \mathrm{i}$ & $0.098208-0.132684 \mathrm{i}$ & $-0.092893-0.054574 \mathrm{i}$ & $-0.089468-0.003980 \mathrm{i}$ \\
\hline 16 & $0.097255-0.133250 \mathrm{i}$ & $0.098903-0.130788 \mathrm{i}$ & $-0.092797-0.055131 \mathrm{i}$ & $-0.089061-0.001961 \mathrm{i}$ \\
\hline 32 & $0.097242-0.133152 \mathrm{i}$ & $0.098895-0.130693 \mathrm{i}$ & $-0.092761-0.055112 \mathrm{i}$ & $-0.089014-0.001963 \mathrm{i}$ \\
\hline 64 & $0.097240-0.133140 \mathrm{i}$ & $0.098894-0.130681 \mathrm{i}$ & $-0.092757-0.055110 \mathrm{i}$ & $-0.089010-0.001963 \mathrm{i}$ \\
\hline 128 & $0.097240-0.133139 \mathrm{i}$ & $0.098894-0.130680 \mathrm{i}$ & $-0.092757-0.055110 \mathrm{i}$ & $-0.089009-0.001963 \mathrm{i}$ \\
\hline
\end{tabular}

$50 \sqrt{3}, \lambda=1$ and the incident angle $\theta=\frac{\pi}{90}$. The interval $[0,2 \pi]$ is divided uniformly for any positive integer $N: t_{j}=j \pi / N, j=0,1, \ldots, 2 N-1$. We choose two points $P_{1}=\left(\frac{\pi}{4}, 2\right), P_{2}=$ $\left(\frac{5 \pi}{4}, 2\right)$ above $\Gamma$ and two points $P_{3}=\left(\frac{3 \pi}{4}, 0\right), P_{4}=\left(\frac{7 \pi}{4}, 0\right)$ below $\Gamma$, and compute the total field $u$ at these four points with different $N$.

The numerical solutions are listed in the following tables, where the exact values are shown in the last line denoted by "exact".

We next present eight numerical examples for periodic profiles: four for the TM-polarization 
Table 7.5: Numerical results for the TM case $\left(\lambda=k_{0}^{2} / k_{1}^{2}\right): k_{0}=50, k_{1}=50 \sqrt{3}, \theta=\frac{\pi}{4}, \Gamma=\Gamma_{2}$.

\begin{tabular}{|c|c|c|c|c|}
\hline$N$ & $u\left(P_{1}\right)$ & $u\left(P_{2}\right)$ & $u\left(P_{3}\right)$ & $u\left(P_{4}\right)$ \\
\hline 128 & $-0.097034-1.119799 \mathrm{i}$ & $-0.167712-1.212785 \mathrm{i}$ & $0.088002-0.796250 \mathrm{i}$ & $-0.805637-0.212324 \mathrm{i}$ \\
\hline 256 & $-0.102483-1.115569 \mathrm{i}$ & $-0.176737-1.206154 \mathrm{i}$ & $0.081493-0.796791 \mathrm{i}$ & $-0.806921-0.206078 \mathrm{i}$ \\
\hline 512 & $-0.102907-1.115214 \mathrm{i}$ & $-0.177469-1.205598 \mathrm{i}$ & $0.081001-0.796823 \mathrm{i}$ & $-0.807000-0.205634 \mathrm{i}$ \\
\hline 1024 & $-0.102864-1.115190 \mathrm{i}$ & $-0.177450-1.205580 \mathrm{i}$ & $0.081071-0.796820 \mathrm{i}$ & $-0.806983-0.205720 \mathrm{i}$ \\
\hline
\end{tabular}

Table 7.6: Numerical results for the TM case $\left(\lambda=k_{0}^{2} / k_{1}^{2}\right): k_{0}=50, k_{1}=50 \sqrt{3}, \theta=\frac{\pi}{90}, \Gamma=\Gamma_{2}$.

\begin{tabular}{|c|c|c|c|c|}
\hline$N$ & $u\left(P_{1}\right)$ & $u\left(P_{2}\right)$ & $u\left(P_{3}\right)$ & $u\left(P_{4}\right)$ \\
\hline 128 & $-1.682832+0.478725 \mathrm{i}$ & $-1.611389+0.604255 \mathrm{i}$ & $-0.039742-0.027628 \mathrm{i}$ & $-0.012424-0.022195 \mathrm{i}$ \\
\hline 256 & $-1.683438+0.482549 \mathrm{i}$ & $-1.608884+0.606089 \mathrm{i}$ & $-0.039754-0.027963 \mathrm{i}$ & $-0.012641-0.022528 \mathrm{i}$ \\
\hline 512 & $-1.683473+0.482879 \mathrm{i}$ & $-1.608669+0.606248 \mathrm{i}$ & $-0.039754-0.027993 \mathrm{i}$ & $-0.012660-0.022557 \mathrm{i}$ \\
\hline 1024 & $-1.683464+0.482884 \mathrm{i}$ & $-1.608681+0.606255 \mathrm{i}$ & $-0.039750-0.027994 \mathrm{i}$ & $-0.012659-0.022556 \mathrm{i}$ \\
\hline
\end{tabular}

Table 7.7: Numerical results for the TE case $(\lambda=1): k_{0}=2, k_{1}=4, \theta=\frac{\pi}{4}, \Gamma=\Gamma_{1}$.

\begin{tabular}{|c|c|c|c|c|}
\hline$N$ & $u\left(P_{1}\right)$ & $u\left(P_{2}\right)$ & $u\left(P_{3}\right)$ & $u\left(P_{4}\right)$ \\
\hline 4 & $-0.810702-0.477550 \mathrm{i}$ & $-0.906885-0.484267 \mathrm{i}$ & $-0.282323+0.181167 \mathrm{i}$ & $-0.116825+0.251700 \mathrm{i}$ \\
\hline 8 & $-0.751113-0.451986 \mathrm{i}$ & $-0.869105-0.488917 \mathrm{i}$ & $-0.374337+0.245366 \mathrm{i}$ & $-0.172844+0.336883 \mathrm{i}$ \\
\hline 16 & $-0.746570-0.448667 \mathrm{i}$ & $-0.862258-0.488053 \mathrm{i}$ & $-0.385146+0.235132 \mathrm{i}$ & $-0.191868+0.324371 \mathrm{i}$ \\
\hline 32 & $-0.746557-0.448689 \mathrm{i}$ & $-0.862238-0.487971 \mathrm{i}$ & $-0.385113+0.235187 \mathrm{i}$ & $-0.191853+0.324295 \mathrm{i}$ \\
\hline 64 & $-0.746555-0.448692 \mathrm{i}$ & $-0.862236-0.487961 \mathrm{i}$ & $-0.385109+0.235194 \mathrm{i}$ & $-0.191850+0.324287 \mathrm{i}$ \\
\hline 128 & $-0.746555-0.448692 \mathrm{i}$ & $-0.862236-0.487960 \mathrm{i}$ & $-0.385109+0.235195 \mathrm{i}$ & $-0.191850+0.324285 \mathrm{i}$ \\
\hline
\end{tabular}

Table 7.8: Numerical results for the TE case $(\lambda=1): k_{0}=2, k_{1}=4, \theta=\frac{\pi}{90}, \Gamma=\Gamma_{1}$.

\begin{tabular}{|c|c|c|c|c|}
\hline$N$ & $u\left(P_{1}\right)$ & $u\left(P_{2}\right)$ & $u\left(P_{3}\right)$ & $u\left(P_{4}\right)$ \\
\hline 4 & $1.155021-0.126414 \mathrm{i}$ & $1.147679-0.132520 \mathrm{i}$ & $-0.014545-0.020530 \mathrm{i}$ & $-0.009546-0.006770 \mathrm{i}$ \\
\hline 8 & $0.961779-0.204560 \mathrm{i}$ & $0.958190-0.210979 \mathrm{i}$ & $-0.030670-0.017289 \mathrm{i}$ & $-0.025319-0.002865 \mathrm{i}$ \\
\hline 16 & $0.956675-0.203385 \mathrm{i}$ & $0.953066-0.209401 \mathrm{i}$ & $-0.030397-0.017575 \mathrm{i}$ & $-0.024978-0.003814 \mathrm{i}$ \\
\hline 32 & $0.956662-0.203404 \mathrm{i}$ & $0.953050-0.209415 \mathrm{i}$ & $-0.030391-0.017588 \mathrm{i}$ & $-0.024967-0.003837 \mathrm{i}$ \\
\hline 64 & $0.956659-0.203404 \mathrm{i}$ & $0.953046-0.209415 \mathrm{i}$ & $-0.030391-0.017590 \mathrm{i}$ & $-0.024966-0.003840 \mathrm{i}$ \\
\hline 128 & $0.956659-0.203404 \mathrm{i}$ & $0.953046-0.209415 \mathrm{i}$ & $-0.030391-0.017590 \mathrm{i}$ & $-0.024966-0.003840 \mathrm{i}$ \\
\hline
\end{tabular}

case (i.e., $\lambda=k_{0}^{2} / k_{1}^{2}$ ) and four for the TE-polarization case (i.e., $\lambda=1$ ). In each polarization case, we use two groups of wave numbers: $k_{0}=2, k_{1}=4$ and $k_{0}=50, k_{1}=50 \sqrt{3}$ and two incident angles: $\theta=\frac{\pi}{4}$ and $\theta=\frac{\pi}{90}$. We will also evaluate the values of the total field at the points $P_{1}, P_{2}, P_{3}, P_{4}$ with different $N$.

In the numerical examples, we consider two grating profiles $\Gamma_{1}$ and $\Gamma_{2}$ given by

$$
\begin{aligned}
& \Gamma_{1}:=\{(t, 1+0.15 \cos 2 t+0.1 \sin t): t \in \mathbb{R}\} \\
& \Gamma_{2}:=\{(t, 1+0.1 \sin t+0.1 \cos 6 t): t \in \mathbb{R}\} .
\end{aligned}
$$

From the numerical results in the above tables it can be concluded that the Nyström method is convergent even for large wave numbers. However, when the wave numbers are getting larger, a much finer mesh grid is needed for a better numerical accuracy. 
Table 7.9: Numerical results for the TE case $(\lambda=1): k_{0}=50, k_{1}=50 \sqrt{3}, \theta=\frac{\pi}{4}, \Gamma=\Gamma_{2}$.

\begin{tabular}{|c|c|c|c|c|}
\hline$N$ & $u\left(P_{1}\right)$ & $u\left(P_{2}\right)$ & $u\left(P_{3}\right)$ & $u\left(P_{4}\right)$ \\
\hline 128 & $-0.050074-0.777549 \mathrm{i}$ & $0.202379-0.941029 \mathrm{i}$ & $0.044722-0.438809 \mathrm{i}$ & $-0.456017-0.115797 \mathrm{i}$ \\
\hline 256 & $-0.047475-0.777410 \mathrm{i}$ & $0.202866-0.943509 \mathrm{i}$ & $0.042104-0.438937 \mathrm{i}$ & $-0.456497-0.113193 \mathrm{i}$ \\
\hline 512 & $-0.047262-0.777401 \mathrm{i}$ & $0.202898-0.943702 \mathrm{i}$ & $0.041890-0.438943 \mathrm{i}$ & $-0.456531-0.112988 \mathrm{i}$ \\
\hline 1024 & $-0.047310-0.777407 \mathrm{i}$ & $0.202886-0.943648 \mathrm{i}$ & $0.041890-0.438946 \mathrm{i}$ & $-0.456531-0.113001 \mathrm{i}$ \\
\hline
\end{tabular}

Table 7.10: Numerical results for the TE case $(\lambda=1): k_{0}=50, k_{1}=50 \sqrt{3}, \theta=\frac{\pi}{90}, \Gamma=\Gamma_{2}$.

\begin{tabular}{|c|c|c|c|c|}
\hline$N$ & $u\left(P_{1}\right)$ & $u\left(P_{2}\right)$ & $u\left(P_{3}\right)$ & $u\left(P_{4}\right)$ \\
\hline 128 & $-0.931555+0.347199 \mathrm{i}$ & $-0.937437+0.346692 \mathrm{i}$ & $-0.012011-0.009864 \mathrm{i}$ & $-0.002162-0.009203 \mathrm{i}$ \\
\hline 256 & $-0.931532+0.347231 \mathrm{i}$ & $-0.937383+0.346765 \mathrm{i}$ & $-0.012005-0.009891 \mathrm{i}$ & $-0.002197-0.009252 \mathrm{i}$ \\
\hline 512 & $-0.931530+0.347233 \mathrm{i}$ & $-0.937378+0.346772 \mathrm{i}$ & $-0.012004-0.009894 \mathrm{i}$ & $-0.002200-0.009256 \mathrm{i}$ \\
\hline 1024 & $-0.931531+0.347234 \mathrm{i}$ & $-0.937379+0.346771 \mathrm{i}$ & $-0.012003-0.009893 \mathrm{i}$ & $-0.002201-0.009256 \mathrm{i}$ \\
\hline
\end{tabular}

\section{Conclusion}

We introduced a new integral equation for the scattering problem from penetrable diffraction gratings (periodic interfaces), which was proved to be equivalent to the scattering problem and uniquely solvable for all but a discrete set of wave numbers. Different from the classical integral equation formulation, the new equation involves only single-layer boundary operators and their normal derivatives which are weakly singular and easy to compute numerically. The Nyström method was then developed for solving the new integral equation effectively, leading to an efficient numerical algorithm for solutions of the scattering problem. Numerical results have also been provided to illustrate the effectiveness of the method. It is remarked that the fast numerical algorithm developed in this paper for the direct scattering problem can be applied to solve the inverse problem of reconstructing the penetrable grating profile from the measured near-field data (see, e.g., $[3,4])$.

Acknowledgments. This work was supported by the NNSF of China under grants 61379093, 91430102, 11401568 and 11501558. The authors thank the anonymous reviewers for their constructive comments and suggestions which helped improve the presentation of the paper.

\section{References}

[1] A. Altundag and R. Kress, On a two-dimensional inverse scattering problem for a dielectric, Appl. Anal., 91 (2012), 757-771.

[2] T. Arens, S.N. Chandler-Wilde and J.A. DeSanto JA, On integral equation and least squares methods for scattering by diffraction gratings, Commun. Comput. Phys., 1:6 (2006), 1010-1042.

[3] G. Bao, P. Li, J. Lin and F. Triki, Inverse scattering problems with multi-frequencies, Inverse Problems, 31:9 (2015), 093001.

[4] G. Bao, A uniqueness theorem for an inverse problem in periodic diffractive optics, Inverse Problems 10 (1994), 335-340.

[5] A. Buffa and R. Hiptmair, Regularized combined field integral equations, Numer. Math., 100 (2005), 1-19. 
[6] S.N. Chandler-Wilde, C.R. Ross and B. Zhang, Scattering by infinite one-dimensional rough surfaces, Proc. Roy. Soc. London, A455 (1999), 3767-3787.

[7] S.N. Chandler-Wilde, E. Heinemeyer and R. Potthast, A well-posed integral equation formulation for three-dimensional rough surface scattering, Proc. Roy. Soc. London, A462 (2006), 3683-3705.

[8] S.N. Chandler-Wilde and B. Zhang, Scattering of electromagnetic waves by rough interfaces and inhomogeneous layers, SIAM J. Math. Anal., 30 (1999), 559-583.

[9] X. Claeys and R. Hiptmair, Multi-Trace boundary integral formulation for acoustic scattering by composite structures, Commun. Pure Appl. Math., 66:8 (2013), 1163-1201.

[10] D. Colton and R. Kress, Integral Equation Methods in Scattering Theory, John Wiley, New York, 1983.

[11] D. Colton and R. Kress, Inverse Acoustic and Electromagnetic Scattering Theory, 3rd ed., Springer, Berlin, 2013.

[12] M. Costabel and E. Stephan, A direct boundary integral equation method for transmission problems, J. Math. Anal. Appl., 106 (1985), 367-413.

[13] J. DeSanto, G. Erdmann, W. Hereman and M. Misra, Theoretical and computational aspects of scattering from periodic surfaces: one-dimensional transmission interface, Waves Random Media, 11 (2001), 425-453.

[14] R. Hiptmair and C. Jerez-Hanckes, Multiple traces boundary integral formulation for Helmholtz transmission problems, Adv. Comput. Math., 37:1 (2012), 39-91.

[15] G.C. Hsiao and R.C. Maccamy, Solution of boundary value problems by integral equations of the first kind, SIAM Review, 15 (1973), 687-705.

[16] G.C. Hsiao and L. Xu, A system of boundary integral equations for the transmission problem in acoustics, Appl. Numer. Math., 61 (2011), 1017-1029.

[17] A. Kirsch, Diffraction by periodic structures, in: Inverse Problems in Mathematical Physics, Lecture Notes in Physics, 422 1993, 87-102.

[18] A. Kirsch, Uniqueness theorems in inverse scattering theory for periodic structures, Inverse Problems, 10 (1994), 145-152.

[19] R.E. Kleimann and P.A. Martin, On single integral equations for the transmission problem of acoustics, SIAM J. Appl. Math., 32:4 (1988), 307-325.

[20] R. Kress, Linear Integral Equations (3rd Edition), Springer, New York, 2014.

[21] R. Kress, On the numerical solution of a hypersingular integral equation in scattering theory, $J$. Comput. Appl. Math., 61 (1995), 345-360.

[22] A. Rathsfeld, G. Schmidt and B.H. Kleemann, On a fast integral equation method for diffraction gratings, Commun. Comput. Phys., 1:6 (2006), 984-1009.

[23] B. Strycharz, Uniqueness in the inverse transmission scattering problem for periodic media, Math. Methods Appl. Sci., 22 (1999), 753-772.

[24] J. Yang and B. Zhang, Uniqueness results in the inverse scattering problem for periodic structures, Math. Methods Appl. Sci., 35 (2012), 828-838.

[25] B. Zhang and S.N. Chandler-Wilde, Integral equation methods for scattering by infinite rough surfaces, Math. Methods Appl. Sci., 26 (2003), 463-488.

[26] H. Zhang and B. Zhang, A novel integral equation for scattering by locally rough surfaces and application to the inverse problem, SIAM J. Appl. Math., 73 (2013), 1811-1829.

[27] R. Zhang and B. Zhang, An FFT-based algorithm for efficient computation of quasi-periodic Green's functions for the Helmholtz and Maxwell equations. Preprint, 2016. 